Fabrication of an ordered nanoparticle array with a nanoaperture membrane used as a contact-mask

This article has been downloaded from IOPscience. Please scroll down to see the full text article. 2006 Nanotechnology 17315

(http://iopscience.iop.org/0957-4484/17/1/054)

The Table of Contents and more related content is available

Download details:

IP Address: 140.112.113.225

The article was downloaded on 21/07/2009 at 09:40

Please note that terms and conditions apply. 


\title{
Fabrication of an ordered nanoparticle array with a nanoaperture membrane used as a contact-mask
}

\author{
Ming-Nung Lin ${ }^{1}$, C Y Liu', N W Liu ${ }^{2,3}$, M Y Lai ${ }^{2}$, C Y Peng ${ }^{1,2}$, \\ H H Wang ${ }^{1,2}$, Y L Wang ${ }^{1,2}$ and Minn-Tsong Lin $^{1,2}$ \\ ${ }^{1}$ Department of Physics, National Taiwan University, Taipei 106, Taiwan \\ ${ }^{2}$ Institute of Atomic and Molecular Sciences, Academia Sinica, PO Box 23-166, Taipei 106, \\ Taiwan \\ ${ }^{3}$ Department of Materials Science and Engineering, National Taiwan University, Taipei 106, \\ Taiwan
}

E-mail: ylwang@pub.iams.sinica.edu.tw and mtlin@phys.ntu.edu.tw

Received 12 July 2005, in final form 10 October 2005

Published 5 December 2005

Online at stacks.iop.org/Nano/17/315

\begin{abstract}
In this paper, we provide a useful technology to fabricate a long-range ordered nanoparticle array with a feature size under $30 \mathrm{~nm}$. By adjusting the incident angle of $\mathrm{Ar}^{+}$beam milling on a U-shaped barrier layer of anodic alumina oxide, we can create a long-range ordered nanoaperture array with samples prepared by a focused-ion-beam-guided process. Compared to the naturally self-organized alumina nanochannels, the FIB-guided process has increased long-range ordering and uniformity of aperture size, and the aperture size can be varied by changing the grazing angle. The nanoaperture membrane can be used as a contact-mask and its undercut structure has another advantage for nanolithography. This technique could be extensively applied to the manufacturing of advanced nanodevices in large areas and as a catalyst to fabricate one-dimensional nanosized materials.
\end{abstract}

(Some figures in this article are in colour only in the electronic version)

\section{Introduction}

Recently, self-organized properties of nanoscale materials have attracted more and more interest [1-3]. One of the important nanostructures is self-organized hexagonally anodic alumina nanochannels. The increasing attraction of porous alumina as a template is due to several advantages, such as its highly uniform and straight nanopore structure, easy and low-cost processing and its extremely high-density advantages. Anodic alumina oxide (AAO) nanochannels are extensively applied to advanced nanodevices such as carbon nanotube arrays for flat panel displays [4], magnetic storage media [5], and single electronics [6]. Besides, long-range order is also another considerable advantage which can be applied to nanolithography. Apart from the fabrication of onedimensional materials, an anodic alumina oxide membrane can be also applied to pattern transfer on a silicon surface.
Some work demonstrated a practical approach of transferring a hexagonal array of porous alumina into silicon substrate [7, 8].

In earlier works, Wang et al $[9,10]$ reported ordered anodic alumina nanochannels on a focused-ion-beam-prepatterned aluminium surface. The ion beam is used to create a hexagonally close-packed lattice of concavities. These concavities act as pinning points to guide the subsequent growth of nanochannels. By carefully matching the lattice constant with the anodization voltage, ordered nanochannels with a high aspect ratio can be fabricated. The pore size of naturally self-organized anodic alumina is determined by the anodization voltage with an appropriate electrolyte, and the proportionality constant of the pore diameter per interpore spacing is usually fixed. For example, the pore diameter is $50 \mathrm{~nm}$ and interpore spacing is $100 \mathrm{~nm}$ with the anodization voltage $40 \mathrm{~V}$ in the oxalic acid solution. Generally speaking, nanopattern feature sizes greater than $100 \mathrm{~nm}$ are routinely 




(b)
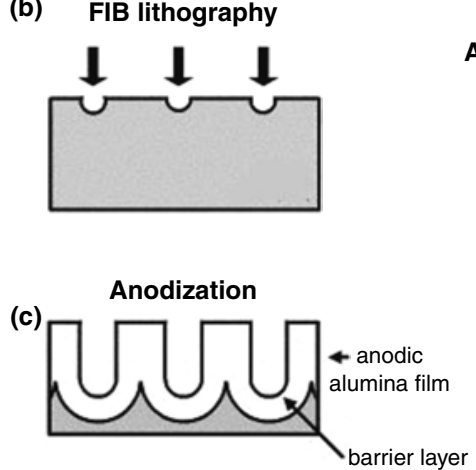

(d)

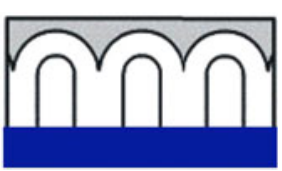

Si substrate

(e)

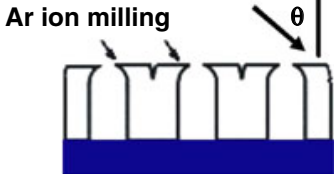

(f)

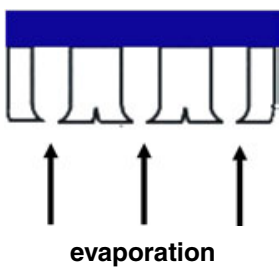

Figure 1. Schematic diagram showing the process for fabrication of the nanopattern apertures.

produced by photolithography techniques. For feature sizes between 30 and $100 \mathrm{~nm}$, electron beam lithography is commonly used. However, feature sizes less than $30 \mathrm{~nm}$ are not easily obtained by standard lithography techniques; therefore, many research groups are actively seeking the way to fabricate AAO with smaller pore size [11-13]. The template synthesis method has played a more and more important role in the fabrication of many kinds of nanostructures, such as quantum dot array, nanowires and nanotubes.

In this work, we will present a combined process of the focused-ion-beam-guided prepatterning technique and grazing $\mathrm{Ar}^{+}$milling process to create a long-range ordered nanoaperture array. This aperture array is of smaller size and the proportionality constant of the aperture diameter per interspacing can be further controlled by the $\mathrm{Ar}^{+}$milling incident angle. The aperture array membrane was thus further used as a lithographic contact-mask to fabricate ordered nanoparticle arrays with different controlled nanoparticle sizes on the silicon surface.

\section{Experimental procedure}

Figure 1 represents the process of the sample preparation. High purity $\mathrm{Al}(99.99 \%)$ was electro-polished in a mixture solution of $\mathrm{HClO}_{4}$ and $\mathrm{C}_{2} \mathrm{H}_{5} \mathrm{OH}$ (ratio 1:5) at $5{ }^{\circ} \mathrm{C}$ under constant stirring (see figure 1(a)). A commercial $50 \mathrm{keV} \mathrm{Ga}$ FIB with beam diameter $\sim 10 \mathrm{~nm}$ and beam current $\sim 1.1 \mathrm{pA}$ was utilized to create an array of hexagonally close-packed concavities on the polished $\mathrm{Al}$ surface (see figure 1(b)). These concavities were employed to guide the anodization growth process of nanochannels. In this work, two samples were prepared with different interspacings. To prepare the first sample with $100 \mathrm{~nm}$ interspacing, we used $0.3 \mathrm{M}$ oxalic acid solution at $4{ }^{\circ} \mathrm{C}$ and $40 \mathrm{~V}$ anodization voltage (see figure $1(\mathrm{c})$ ).

The remaining $\mathrm{Al}$ substrate is removed by a $\mathrm{CuCl}_{2}$ solution

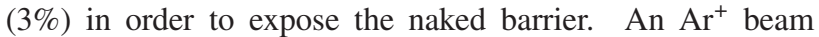
miller machine MPS-3000 PBN was set up to sputter the U-shaped barrier layer in order to create the nanoapertures (see figure 1(e)). A detailed description of the fabrication of long-range ordered nanoapertures can also be found in our recent work [14]. The $\mathrm{Ar}^{+}$beam current density was approximately $1.2 \mathrm{~mA} \mathrm{~cm}{ }^{-2}$ with beam energy $500 \mathrm{eV}$. The incident $\mathrm{Ar}^{+}$beam was tilted by an angle $\theta=82^{\circ}(\theta$ is defined as the angle between the incident direction and the normal axis of the plane) to control the aperture size. Finally, the images of nanoapertures were taken by a transmission electron microscope (TEM). By taking the top view of the thin $(\sim 150 \mathrm{~nm})$ alumina film, the electrons directly passing through the apertures without any interceptions will project these apertures to form the bright spot image on the screen. To prepare the second sample with $50 \mathrm{~nm}$ interspacing, we used the same oxalic acid solution at $4{ }^{\circ} \mathrm{C}$ but different anodization voltage $(20 \mathrm{~V})$. The incident $\mathrm{Ar}^{+}$beam was tilted by an angle of around $75^{\circ}$ to create the same aperture size as the first sample. The images of these nanoapertures were also taken by TEM. By mounting a nanoaperture array membrane on the silicon substrate (see figure 1(d)), this membrane was used as a contact-mask to fabricate gold nanoparticles. The gold particles are thermally evaporated through the nanoapertures and finally deposited onto the substrate (see figure 1(f)). The scanning electron microscope (SEM) was employed to take images of the nanoparticle array.

\section{Results and discussion}

Figure 2(a) represents the TEM image with a boundary between the two areas: naturally self-organized growth mode (left side) and FIB-guided growth mode (right side). It is clear to see the difference between the two various growth modes. The uniformity for both interspacing and aperture size in the FIB-guided growth mode is much better than that in the naturally self-organized growth mode. Fourier transform was made for each area in order to demonstrate the differences in long-range ordering between the two growth modes. In the self-organized area, the emerging peaks of the Fourier transform form an isotropic ring pattern, indicating the absence of long-range ordering. In the FIB-guided area, the hexagonally arranged peaks become sharper. This indicates the marked improvement of the degree of order in the orientation arrangement of the nanoapertures. Figures 2(b) and (c) show the TEM images of the nanoapertures in a FIBguided area on different scales. These apertures are mostly of a round shape, and reveal a uniform size distribution with a nominal diameter around $12 \mathrm{~nm}$. Moreover, the interspacing between these neighbouring apertures also has the value of $100 \mathrm{~nm}$. The electrons passing through these apertures were indeed exposed to form the bright spots in the TEM images. These bright spots in the TEM image are the projections of the transmitted electron beam through the whole channels. Both the roughness of the channel wall and the inclined angle of the pore will affect the projection image of the electron beam. Thus, the nominal diameter of the apertures obtained from the TEM image also reflects the overall effect of geometrical factors. The TEM images clearly show that the aperture array 
(a)

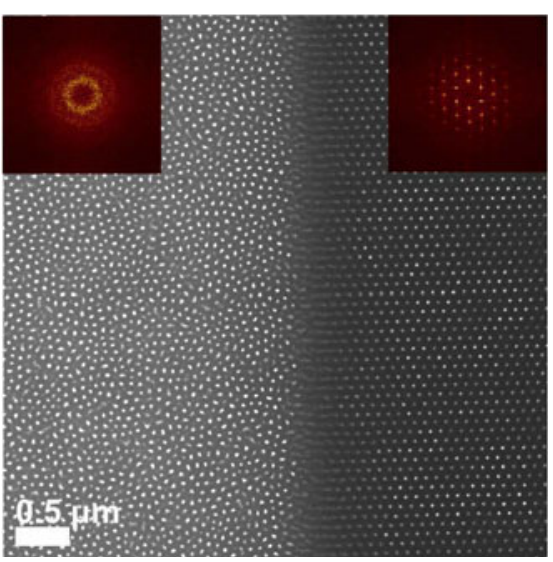

(b)

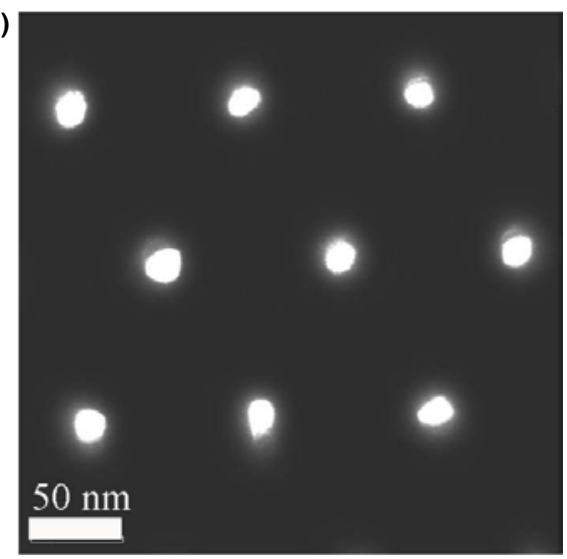

(c)

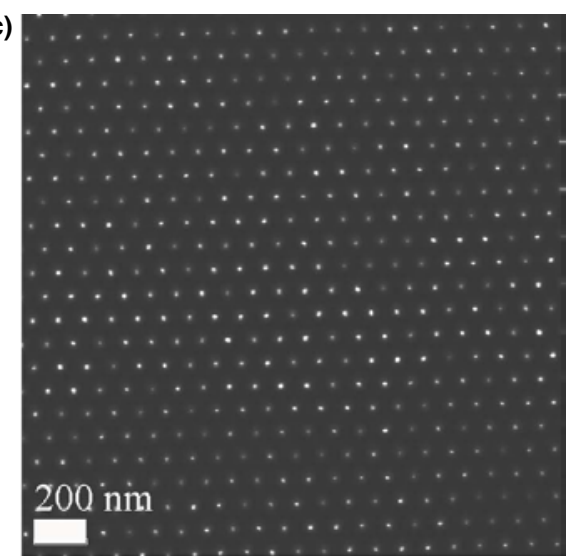

(d)

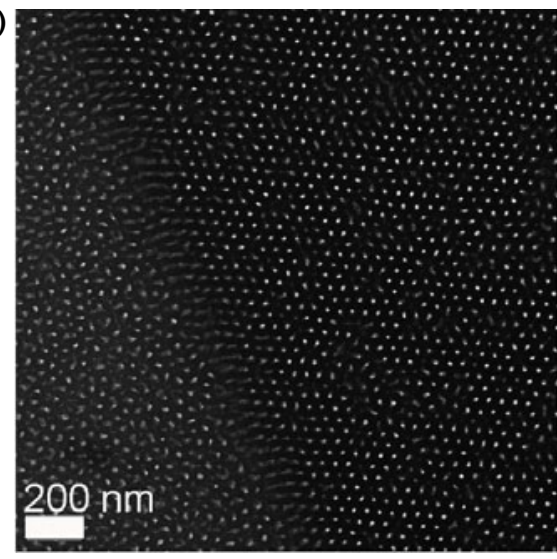

(e)



(f)

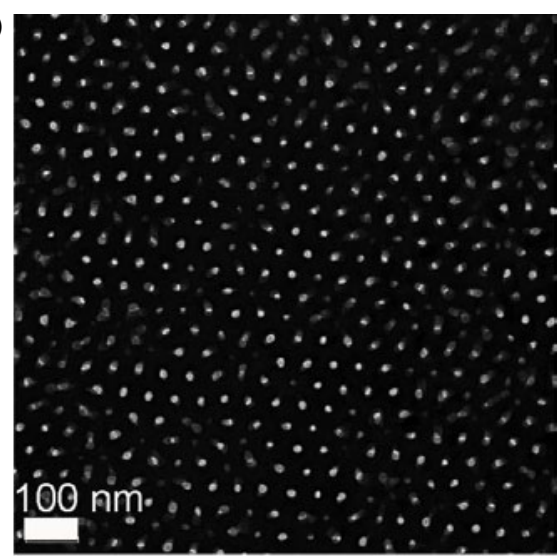

Figure 2. TEM image in the FIB-guided area. (a)-(c) TEM image of the aperture array in different scales with $100 \mathrm{~nm}$ interspacing; (d)-(f) TEM image of the aperture array in different scales with $50 \mathrm{~nm}$ interspacing.

is an undercut nanostructure with highly straight pores. These advantages of the special nanostructure type will be very useful for application to a lithographic contact-mask. Furthermore, we varied two factors (interspacing and incident angle) to control the same aperture size.

Figures 2(d)-(f) are TEM images of the aperture array in different scales with $50 \mathrm{~nm}$ interspacing (second sample). The incident angle of the $\mathrm{Ar}^{+}$beam was tilted by $75^{\circ}$ in order to create the same aperture size as the first sample. The proportionality constant of pore diameter per interspacing is usually limited and inalterable for the naturally self-organized AAO. Now, we can choose a variable proportionality constant by selecting the appropriate incident angle. The uniformity of nanoapertures in the second sample is not as good as the first sample. It seems rather difficult to fabricate long-range ordered nanochannels with a pore size less than $30 \mathrm{~nm}$ for the natural limit in the FIB-guided grown process. However, the uniformity of pore size of nanochannels will influence the uniformity of nanoaperture size; therefore, this limit will also affect the uniformity of created nanoapertures.

Figure 3 is a statistical analysis in the geometrical distribution made in order to show the uniformity of nanoapertures. We randomly chose 100 apertures to measure the diameter size of the aperture. The size distribution of 
(a)

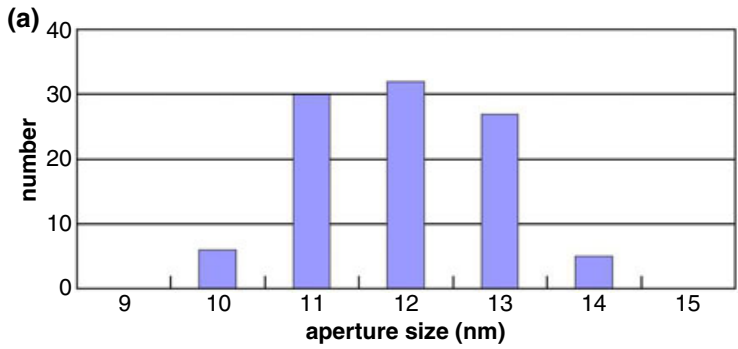

(c)



(b)

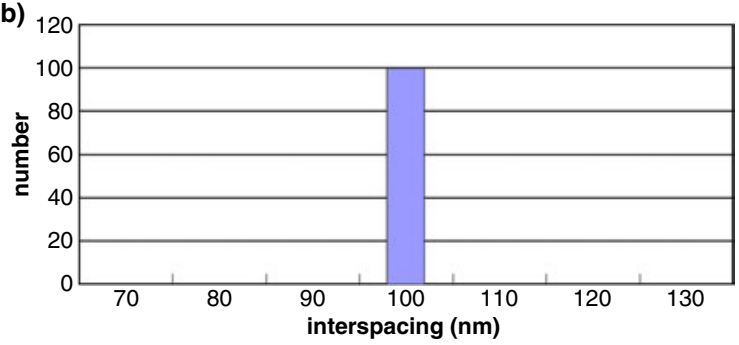

(d)

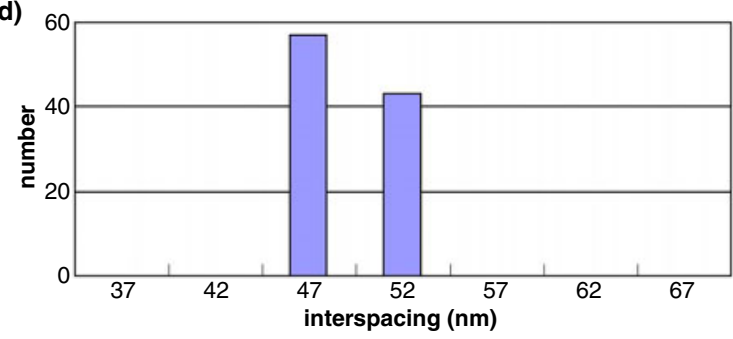

Figure 3. The statistical analysis for two samples: (a) aperture size distribution of the first sample; (b) interspacing distribution of the first sample; (c) aperture size distribution of the second sample; (d) interspacing distribution of the second sample.

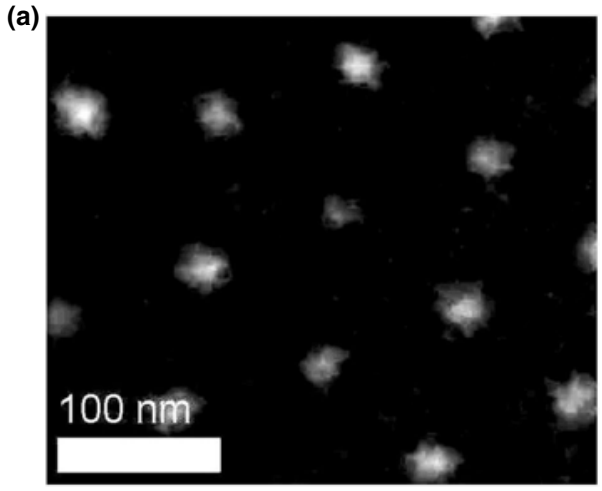

(b)

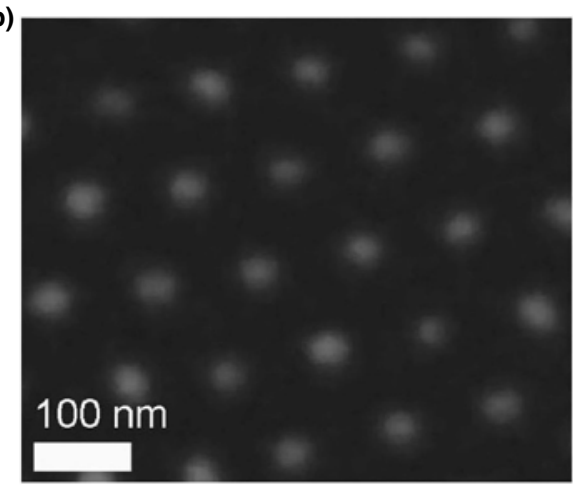

(c)



(d)



Figure 4. SEM image of the nanoparticle array with an aperture membrane used as a contact-mask; the mean size of the nanoparticles is controlled by the grazing angle $\theta$ of the $\mathrm{Ar}^{+}$beam: (a) $\theta=60^{\circ}$; (b) $\theta=75^{\circ}$; (c) $\theta=82^{\circ}$. (d) Relation diagram between the nanoparticle size and $\cos \theta$.

nanoapertures for the first sample is shown in figure 3(a). The mean value of the FIB-guided aperture size is $12 \mathrm{~nm}$ with dispersion $\pm 2 \mathrm{~nm}$. More than $80 \%$ of aperture sizes are distributed between 11 and $13 \mathrm{~nm}$. The interspacing distribution of nanoapertures is shown in figure $3(b)$. The mean value of the interspacing is $100 \mathrm{~nm}$ with dispersion of $\pm 2 \mathrm{~nm}$. Relatively, the average size of the apertures made from the self-organized AAO is $13 \mathrm{~nm}$, but with much larger dispersion of $\pm 6 \mathrm{~nm}$. These differences can be ascribed to two factors. First, the aperture size depends on the pore size and curvature of the barrier layer with the same inclined grazing $\mathrm{Ar}^{+}$milling angle. The size distribution and the interspacing between neighbouring channels were demonstrated to be excellently uniform in the FIB-guided grown sample [9]. In contrast, the naturally self-organized sample cannot achieve such uniformity. Second, the relative 
height around neighbouring channels also affects the aperture size. The lower channel (compared to nearby channels) will be shielded by its neighbours under the inclined grazing $\mathrm{Ar}^{+}$ beam. This will result in a smaller aperture. Compared to the self-organized mode, the growth rates and heights of each channel of the FIB-guided sample are more alike. This also improves the uniformity of the aperture size.

The size and interspacing distribution of nanoapertures (second sample) are shown in figures $3(\mathrm{c})$ and (d). The mean value of the aperture size is $10 \mathrm{~nm}$ with dispersion $\pm 2 \mathrm{~nm}$. The mean value of the interspacing is $50 \mathrm{~nm}$ with dispersion of $\pm 3 \mathrm{~nm}$. This result demonstrates that we can control the same aperture size by adjusting the interspacing and incident angle.

Finally, we will demonstrate a convenient way to fabricate the ordered nanoparticle array. By mounting a nanoaperture array membrane on the silicon substrate, the hexagonally close-packed lattice pattern of anodic alumina can be precisely transferred onto the silicon surface. With the nanoaperture array membrane as a contact-mask, gold particles are evaporated through the nanoapertures and deposited onto the silicon surface. As shown in figure 4, the mean size of the nanoparticles is determined by the mean size of the nanoapertures; however, the mean size of the nanoapertures is controlled by the inclined grazing angle of the $\mathrm{Ar}^{+}$beam. With the inclined grazing angle $\theta=60^{\circ}, 75^{\circ}$, and $82^{\circ}$, the mean size of the nanoparticles is 12,25 , and $40 \mathrm{~nm}$, respectively. The interspacing is fixed at $100 \mathrm{~nm}$ in these samples. In figure 4 , the mean size of the nanoparticles is roughly proportional to $\cos \theta$. This indicates that the aperture size is determined by the projection area of the U-shape barrier layer. Only the area exposed to the $\mathrm{Ar}^{+}$beam will be removed. The sputtering time is different for the different sputtering rates with different incident angles. Thus, it is convenient to fabricate variable sizes of nanoparticle by just selecting the appropriate incident angle. In general, the size of the nanoparticle array fabricated by e-beam lithography or a photolithography system depends on the beam dosage and the properties of the resists. These factors are rather complicated and usually difficult to control well. There is also an advantage in our case that the aperture size is smaller than the pore size.
As mentioned above, the TEM images show that the aperture array membrane forms an undercut nanostructure with highly straight pores. This geometric advantage is important for the following lift-off process. The undercut structure will prevent the damage of the pattern which is often caused in the lift-off process.

\section{Conclusion}

In summary, with grazing $\mathrm{Ar}^{+}$milling on the U-shaped barrier layer of AAO grown by the FIB guided process, we can fabricate an aperture array with a controlled diameter of around $10 \mathrm{~nm}$. The Fourier transform of the array image reveals a hexagonal symmetry. The interspacing is defined by the focused ion beam lithography. The aperture sizes can be controlled by different incident angles of the $\mathrm{Ar}^{+}$ beam. This aperture array membrane can be further applied to a lithographic contact-mask for fabrication of an ordered nanoparticle array $[4,5,7,8]$.

\section{References}

[1] Wilbur J L and Whitesides G M 1999 Self-assembly and self-assembled monolayers in micro- and nanofabrication Nanotechnology ed G Timp (Berlin: Springer) p 331

[2] Lin W-C, Kuo C-C, Luo M-F, Song K-J and Lin M-T 2005 Appl. Phys. Lett. 86043105

[3] Gwo S, Chou C-P, Wu C-L, Ye Y-J, Tsai S-J, Lin W-C and Lin M-T 2003 Phys. Rev. Lett. 90185506

[4] Suh J S and Lee J S 1999 Appl. Phys. Lett. 752047

[5] Nielsch K, Wehrspohn R B, Barthel J, Kirschner J and Gösele U 2001 Appl. Phys. Lett. 791360

[6] Kouklin N and Menon L 2002 Appl. Phys. Lett. 801649

[7] Crouse D and Lo Y-H 2000 Appl. Phys. Lett. 7649

[8] Kanamori Y and Hane K 2001 Appl. Phys. Lett. 78142

[9] Liu C Y, Datta A and Wang Y L 2001 Appl. Phys. Lett. 78120

[10] Liu C Y, Datta A, Liu N W, Peng C Y and Wang Y L 2004 Appl. Phys. Lett. 842509

[11] Li A P, Müller F, Birner A, Nielsch K and Gösele U 1998 J. Appl. Phys. 846023

[12] Xu T, Zangari G and Metzger R M 2002 Nano. Lett. 2 37-41

[13] Asoh H and Nishio K 2001 J. Vac. Sci. Technol. B 19569

[14] Lin M N, Lin M-T, Liu C Y, Lai M Y, Liu N W, Peng C Y, Wang H H and Wang Y L 2005 Appl. Phys. Lett. 87173116 